\title{
SEGRE NONDEGENERATE TOTALLY REAL SUBVARIETIES
}

\author{
BERNHARD LAMEL AND JIŘÍ LEBL
}

\begin{abstract}
We study an irreducible real-analytic germ of an $n$-dimensional variety in $n$ dimensional complex space. Assuming that the variety is Segre nondegenerate we define an averaging operator that generalizes the MoserWebster involution. This operator can be thought of as being the CR structure of the singularity, and using this operator we study the set of functions that are restrictions of holomorphic functions. We give a condition on the flattening of the singularity, that is realizing the singularity as a codimention one subvariety of a nonsingular Levi-flat hypersurface.
\end{abstract}

\section{INTRODUCTION}

A natural question in complex analysis is the following:

Given a set $X \subset \mathbb{C}^{n}$, characterize those functions $f: X \rightarrow \mathbb{C}$ that are restrictions of holomorphic functions defined in a neighborhood of $X$.

When $X$ is a real-analytic CR submanifold, then the answer is well-understood, it is the set of real-analytic CR functions, that is functions that satisfy the CauchyRiemann equations restricted to $X$. If $X$ is a generic $n$-dimensional submanifold, such as $X=\mathbb{R}^{n} \subset \mathbb{C}^{n}$, then the CR structure of $X$ is trivial, and every real-analytic function is the restriction of a holomorphic function.

If $X$ is singular, then the answer is much more difficult. The case that we are interested in is the local question near a singular point of a real-analytic subvariety of dimension $n$. At a nondegenerate singular point we will be define a finite alternative to the Cauchy-Riemann equations. The CR equations normally say that a holomorphic function is constant along a certain (complex) direction. In our setting, we will replace this complex direction with a finite set of points. The setting applies also to CR singular manifolds, and given a Bishop surface, that is a 2 dimensional real submanifold of $\mathbb{C}^{2}$ with a nondegenerate complex tangent, see [2]. This finite set of points are precisely the two from the Moser-Webster involution, see [18. In $\mathbb{C}^{2}$, the CR singular case, focused mainly on normal forms, was studied further by Moser [17, Kenig-Webster [13, Harris [7, Gong [4, Huang-Krantz 9], HuangYin [12, and others. Harris 8 studied the restriction question on a CR singular submanifold in terms of vector fields defined on $M$. Lebl-Noell-Ravisankar [15] proved that functions satisfying a moment condition on the elliptic Bishop surface are restrictions of holomorphic functions. CR singular submanifolds of dimension $n$ have similarly been studied by Webster [19, Kenig-Webster [14, Huang [10, Coffman [3, Ahern-Gong [1, Gong-Stolovich [5, 6] and others. See also the survey by Huang [11].

Date: January 24, 2020.

2010 Mathematics Subject Classification. 32V05,32V40,14B05,14P15. 
The Segre variety of an $n$-dimensional real subvariety of $\mathbb{C}^{n}$ is generically a finite set of points. As one can average over a finite set of points, we obtain an operator from the real power series $\mathbb{C}\{z, \bar{z}\}$ to the holomorphic power series $\mathbb{C}\{z\}$. The operator is given explicitly, and can be computed for a given subvariety up to any given order. The operator reproduces the holomorphic functions, and therefore can be used to answer the motivating question: What are the restrictions of holomorphic functions?

The operator can also be used to attempt to find normal forms for $X$. As $\mathbb{R}^{n}$ is given by the vanishing of the imaginary part of $n$ independent holomorphic functions, one can similarly ask to find at least one such function for a singular $X$. Such a function we call a "flattening", as it gives a Levi-flat hypersurface that contains $X$. The flattening question is equivalent to finding a holomorphic function $f$ such that $\bar{f}=f$ on $X$, or in other words when the averaging operator is applied to $\bar{f}$ it simply yields $f$. As the operator works formally, one can use it to explicitly find obstructions to flattening.

The flattening question only requires the restriction of the averaging operator to $\mathbb{C}\{\bar{z}\}$. It turns out that this restriction uniquely describes the germ of $X$ at a point. That is, finding local normal forms for $X$ is equivalent to finding the normal forms for the restricted operator.

Let us state our main results more precisely. Let $(X, 0) \subset\left(\mathbb{C}^{n}, 0\right)$ be an (irreducible) $n$-dimensional germ of a real-analytic subvariety at the origin in $\mathbb{C}^{n}$. We denote by $\mathcal{I}_{0}(X) \subset \mathbb{C}\{z, \bar{z}\}$ the ideal of germs of real-analytic functions vanishing on $X$. We say that $(X, 0)$ is Segre nondegenerate if the ideal $\mathcal{J}=\left\{\varrho(z, 0): \varrho \in \mathcal{I}_{0}(X)\right\} \subset$ $\mathbb{C}\{z\}$ is an ideal of definition, i.e. if there exists a $k \in \mathbb{N}$ such that the maximal ideal $\mathfrak{m} \subset \mathbb{C}\{z\}$ satisfies $\mathfrak{m}^{k} \subset \mathcal{J}$, or equivalently, if its vanishing locus satisfies $V(\mathcal{J})=\{0\}$.

Let $\mathcal{O}_{0}$ be the ring of germs of holomorphic functions. We say a germ $(f, 0)$ of a function is real-analytic on $(X, 0)$ if there is a germ of a real-analytic function defined in $\left(\mathbb{C}^{n}, 0\right)$ whose restriction to $(X, 0)$ is $(f, 0)$. In this case we will often identify $(f, 0)$ with its extension. We say $(f, 0)$ is a restriction of a holomorphic function if there exists an extension such that $(f, 0) \in \mathcal{O}_{0}$.

To make notation easier, we will sometimes drop the $(\cdot, 0)$ notation when not absolutely necessary for clarity. Given a small enough neighborhood, a real-analytic function has a unique representative and we will generally identify the germ with one of its representatives.

Let $(X, 0) \subset \mathbb{C}^{n}$ be an irreducible germ of a Segre nondegenerate $n$-dimensional real-analytic subvariety of multiplicity $k$. That is, the Segre variety generically has $k$ points. Given a real-analytic function $f(z, \bar{z})$ we let $\mathcal{A} f$ be the average $\frac{1}{k} \sum_{j} f\left(z, \xi_{j}\right)$ over the points $\xi_{1}, \ldots, \xi_{k}$ of the Segre variety at $z$.

Our first main result is that $f$ is a restriction of a holomorphic function if and only if

$$
\mathcal{A}\left(f^{\ell}\right)=(\mathcal{A} f)^{\ell} \text { for all } \ell=1, \ldots, k \text {. }
$$

Let us call $\mathcal{R}$ the restriction of $\mathcal{A}$ to the antiholomorphic functions. It turns out that $\mathcal{R}$ contains all the information about $(X, 0)$ and $\mathcal{A}$, that is, finding a normal form for $\mathcal{R}$ is equivalent to finding a normal form for $(X, 0)$.

A natural question about the normal form of $(X, 0)$ is the so-called flattening. That is, does there exist a holomorphic function that is real-valued on $(X, 0)$. We 
prove that a holomorphic function $f$ is real-valued on $X$ if and only if

$$
\mathcal{R}\left(\bar{f}^{\ell}\right)=f^{\ell} \text { for all } \ell=1, \ldots, k .
$$

Let us outline the structure of this paper. In $\$ 2$, we explain the notation and the setup of the problem including complexification and the Segre varieties. In 93. we study the obstructions for a function to be the restriction of a holomorphic function. In $\$ 4$ we define the averaging operator. In \$5, we discuss the restricted averaging operator, and its application to flattening and show that it contains all the necessary information to define $X$. In $₫ 6$, we work out the flattening in some examples.

\section{Preliminaries}

We start with some properties of the extrinsic complexification of a Segrenondegenerate germ: we recall that $(\mathcal{X}, 0) \subset\left(\mathbb{C}_{z, \xi}^{2 n}, 0\right)$ is a complexification of $(X, 0)$ if $\mathcal{X} \cap\{z=\bar{\xi}\}$ as germs at the origin. We denote the projections onto the first and the second factor by $\pi_{1}(z, \xi)=z$ and $\pi_{2}(z, \xi)=\xi$, respectively.

Proposition 2.1. Let $(X, 0) \subset\left(\mathbb{C}_{z}^{n}, 0\right)$ be a germ of a Segre nondegenerate irreducible $n$-dimensional subvariety at the origin. Then there exist polydiscs $\Delta_{z} \subset \mathbb{C}^{n}$ and $\Delta_{\xi} \subset \mathbb{C}^{n}$, centered at the origin, and an n-dimensional closed complex subvariety $\mathcal{X} \subset \Delta_{z} \times \Delta_{\xi}$ which is irreducible both globally and at the origin, such that $(\mathcal{X}, 0)$ is the complexification of $(X, 0)$, and an integer $k$, such that $\left.\pi_{1}\right|_{\mathcal{X}}$ is finite, and $\left(\pi_{1} \mid \mathcal{X}\right)^{-1}(z)$ consists of $k$ points counting multiplicity for $z \in \Delta_{z}$, and furthermore $\left(\pi_{1} \mid \mathcal{X}\right)^{-1}(0)=\{0\}$.

The same statement holds with a possibly different pair of polydiscs $\tilde{\Delta}_{z} \subset \mathbb{C}^{n}$ and $\tilde{\Delta}_{\xi} \subset \mathbb{C}^{n}$ and the projection onto the second coordinate $\pi_{2}$.

Definition 2.2. If $(X, 0) \subset\left(\mathbb{C}^{n}, 0\right)$ is a germ at 0 of a Segre nondegenerate $n$ dimensional subvariety, then we call the polydiscs $\Delta_{z} \times \Delta_{\xi} \pi_{1}$-good for $(X, 0)$, if they are small enough as above, and we will call the closed subvariety $\mathcal{X} \subset \Delta_{z} \times \Delta_{\xi}$ the corresponding complexification; the polydiscs $\tilde{\Delta}_{z} \times \tilde{\Delta}_{\xi}$ will be said to be $\pi_{2}$-good, and the corresponding complexification is defined likewise. We will call $k$ the Segre multiplicity of $(X, 0)$.

The germ of $\mathcal{X}$ at the origin is well defined, and for small neighbourhoods the corresponding complexification is just a representative of that germ in that neighbourhood. We will therefore use $\mathcal{X}$ as a notation for the complexification in any (small enough) neighbourhood.

Proof. We only the prove the corresponding statement for the $\pi_{1}$-good polydiscs; the $\pi_{2}$-good polydiscs are done analogously. First we find a small enough neighborhood of the origin and a complexification that is irreducible at the origin. That follows by simply taking the smallest germ of a complex subvariety that contains the germ of the set

$$
\{(z, \xi): \bar{z}=\xi, z \in X\}
$$

at the origin, for some representative $X$ of $(X, 0)$. If $\mathcal{X}$ were not irreducible, it would imply $(X, 0)$ is also reducible by restricting the components of $\mathcal{X}$ to the diagonal $\bar{z}=\xi$. Making the neighborhood $\Delta_{z} \times \Delta_{\xi}$ small enough we can ensure that the complexification is irreducible for any other smaller polydisc neighborhood. 
The subvariety $X$ is $n$-real-dimensional, and hence $\mathcal{X}$ is $n$-complex-dimensional. Because $X$ is Segre nondegenerate, then the $n$-dimensional subspace $\{0\} \times \mathbb{C}^{n} \subset$ $\mathbb{C}^{n} \times \mathbb{C}^{n}$ intersects $\mathcal{X}$ at an isolated point at the origin. In particular, this means that locally near the origin, $\mathcal{X}$ is a multigraph of a $k$-valued holomorphic mapping (see [20]). We can now choose $\Delta_{z}$ small enough that $\left(\pi_{1} \mid \mathcal{X}\right)^{-1}$ has generically $k$ preimages in $\Delta_{\xi}$ (exactly $k$ counting multiplicity), and furthermore that $\left(\pi_{1} \mid \mathcal{X}\right)^{-1}(0)$ is the origin alone.

In terms of the ideal $\mathcal{I}_{0}(X) \subset \mathbb{C}\{z, \bar{z}\}$ of $(X, 0)$, i.e. the set of germs of realanalytic functions at 0 vanishing on $X$, we have that $\mathcal{I}_{0}(X)$ is a real ideal. That is, $\iota\left(\mathcal{I}_{0}(X)\right) \subset \mathcal{I}_{0}(X)$, where $(\iota \varrho)(z, \bar{z})=\bar{\varrho}(\bar{z}, z)$. One can check that the ideal of any complexification $\mathcal{X}$ (in either a $\pi_{1}$ - or a $\pi_{2}$-good polydisc) at the origin is given by $\mathcal{I}_{0}(\mathcal{X})=\left\{\varrho(z, \xi): \varrho(z, \bar{z}) \in \mathcal{I}_{0}(X)\right\}$. Now, because $\mathcal{I}_{0}(X)$ is real, we have that the involution $\iota: \mathbb{C}\{z, \xi\} \rightarrow \mathbb{C}\{z, \xi\}$ defined by $\iota(\varrho)(z, \xi)=\bar{\varrho}(\xi, z)$ leaves $\mathcal{I}_{0}(\mathcal{X})$ invariant. For a set $A \subset \mathbb{C}^{n}$ we are going to denote the by $A^{*}$ the set of complex conjugates of elements of $A$. The preceding algebraic fact has the following geometric interpretation:

Proposition 2.3. Let $(X, 0) \subset\left(\mathbb{C}_{z}^{n}, 0\right)$ be a germ of a Segre nondegenerate irreducible $n$-dimensional subvariety at the origin, $\mathcal{X}$ a complexification of $X$. Then for a small enough neighbourhood $\Delta$ of the origin, the map $S: \Delta \times \Delta^{*} \rightarrow \Delta \times \Delta^{*}$ defined by $S(z, \xi)=(\bar{\xi}, \bar{z})$ leaves $\mathcal{X} \cap\left(\Delta \times \Delta^{*}\right)$ invariant.

Proof. We can choose real generators of $\mathcal{I}_{0}(\mathcal{X})$, i.e. germs $\varrho_{1}, \ldots, \varrho_{p} \in \mathbb{C}\{z, \xi\}$ satisfying $\varrho_{j}(z, \xi)=\bar{\varrho}_{j}(\xi, z)$ for $j=1, \ldots, p$. Now, for $z$ and $\xi$ sufficiently close to $0,(z, \xi) \in \mathcal{X}$ if and only if $\varrho_{j}(z, \xi)=0$ for all $j$, which in turn is equivalent to $\bar{\varrho}_{j}(\bar{z}, \bar{\xi})=\varrho_{j}(\bar{\xi}, \bar{z})=0$ for $j=1, \ldots, p$, i.e. $(\bar{\xi}, \bar{z}) \in \mathcal{X}$.

Proposition 2.3 allows us to identify representatives of $(X, 0)$ with the diagonal $z=\bar{\xi}$ in $\mathcal{X} \cap\left(\Delta \times \Delta^{*}\right)$ for small polydiscs $\Delta$. Whenever we need to refer to a representative of the germ $(X, 0)$, we will choose one of the form constructed in this proposition.

Proposition 2.4. Let $(X, 0)$ is an irreducible $n$-dimensional germ of a real subvariety at the origin. If $(X, 0)$ is Segre nondegenerate, then at a generic dimension-n regular point of a small enough representative, $X$ is a maximally totally real submanifold. Furthermore, the germ $(X, 0)$ is not contained in any germ of a proper complex analytic subvariety at the origin.

Proof. Let $\Delta_{z} \times \Delta_{\xi}$ be good for $(X, 0)$ and let $\mathcal{X}$ be the corresponding complexification. The projection $\left(\pi_{1} \mid \mathcal{X}\right)$ is generically $k$-to- 1 . If $(X, 0) \subset(Y, 0)$ for a germ of a proper complex analytic subvariety then $\left(\pi_{1} \mid \mathcal{X}\right)^{-1}(z)$ would be empty for $z \notin Y$. Hence, $(X, 0)$ is not contained in any proper complex subvariety.

Since the discriminant set of $\left.\pi_{1}\right|_{\mathcal{X}}$ is a complex subvariety in $\Delta_{z}$, at a generic point $z_{0} \in X$ we have that $\left(z_{0}, \bar{z}_{0}\right)$ is a regular point of $\mathcal{X}$, and hence $z_{0}$ is a regular point of $X$. Then locally near $\left(z_{0}, \bar{z}_{0}\right), \mathcal{X}$ can be written as a graph $\xi=g(z)$, and hence $X$ near $z_{0}$ is given by the (vector) equation $\bar{z}=g(z)$. In other words $X$ near $z_{0}$ is a maximally totally real submanifold.

2.1. Symmetric functions and standard defining equations. We will now recall some standard facts about analytic varieties from Whitney's book [20] in the setting we need. 
If $(X, 0)$ is a Segre nondegenerate germ of multiplicity $k, \tilde{\Delta}_{z} \times \tilde{\Delta}_{\xi}$ is $\pi_{2}$-good for $X$, and $\mathcal{X}$ is the corresponding complexification, then one can consider the $k$ (generically distinct) points $\alpha^{1}(\xi), \ldots, \alpha^{k}(\xi) \in \tilde{\Delta}_{z}$, defined for $\xi \in \tilde{\Delta}_{\xi}$, satisfying $\left(\alpha^{j}(\xi), \xi\right) \in \mathcal{X}$ for $j=1, \ldots, k$ as a point $Z(\xi)=\left\langle\alpha^{1}(\xi), \ldots, \alpha^{k}(\xi)\right\rangle \in\left(\mathbb{C}^{n}\right)_{\text {sym }}^{k}$.

Here $X_{\text {sym }}^{k}$ is the $k$-th symmetric power of $X$, i.e. the quotient of $X^{k}$ with respect to the equivalence relation identifying two points $\left(\alpha^{1}, \ldots, \alpha^{k}\right)$ with $\left(\beta^{1}, \ldots, \beta^{k}\right)$ if $\beta^{j}=\alpha^{\ell_{j}}$ for some permutation $j \mapsto \ell_{j}$ of $\{1, \ldots, k\}$. We shall write $\alpha \in\left\langle\alpha^{1}, \ldots, \alpha^{k}\right\rangle$ if $\alpha=\alpha^{j}$ for some $j$.

The variety $\mathcal{X}$ can be considered as the multigraph of the holomorphic multifunction $Z(\xi)$ in $\Delta_{z} \times \Delta_{\xi}$, meaning that for any symmetric function $h:\left(\mathbb{C}^{n}\right)^{k} \rightarrow \mathbb{C}$ the composition

$$
h(Z(\xi))=h\left(\alpha^{1}(\xi), \ldots, \alpha^{k}(\xi)\right) \in \mathcal{H}\left(\Delta_{\xi}\right)
$$

is holomorphic in $\Delta_{\xi}$.

The elementary symmetric functions on $\left(\mathbb{C}^{n}\right)^{k}$ are the coefficients $\phi_{\ell, \gamma}$ of the polynomial

$$
P_{n, k}(x, u)=\prod_{j=1}^{k}\left(x-u \cdot \alpha^{j}\right)=\sum_{\ell=0}^{k}\left(\sum_{\substack{\gamma \in \mathbb{N}^{n} \\|\gamma|=\ell}} \phi_{\ell, \gamma}\left(\alpha^{1}, \ldots, \alpha^{k}\right) u^{\gamma}\right) x^{k-\ell} .
$$

In terms of the elementary symmetric functions $P_{\ell, k} \in \mathbb{C}\left[y^{1}, \ldots y^{k}\right]$, where the polynomial $P_{\ell, k}\left(y^{1}, \ldots, y^{k}\right)$ is defined by

$$
\prod_{j=1}^{k}\left(x-y^{j}\right)=\sum_{\ell=0}^{k} P_{\ell, k}\left(y^{1}, \ldots, y^{k}\right) x^{k-\ell},
$$

they can also be expressed through the coefficients of the polynomials

$$
P_{\ell, k}\left(u \cdot \alpha^{1}, \ldots, u \cdot \alpha^{k}\right)=\sum_{\substack{\gamma \in \mathbb{N}^{n} \\|\gamma|=\ell}} \phi_{\ell, \gamma}\left(\alpha^{1}, \ldots, \alpha^{k}\right) u^{\gamma}
$$

In order to transfer results for symmetric functions of $k$ scalar variables to the multivariate case, note that for any $u \in \mathbb{C}^{n}$, and any symmetric polynomial $P\left(y^{1}, \ldots, y^{k}\right)$ we have that $P\left(u \cdot \alpha^{1}, \ldots, u \cdot \alpha^{k}\right)$ is a symmetric function of $\left(\alpha^{1}, \ldots, \alpha^{k}\right) \in\left(\mathbb{C}^{n}\right)^{k}$. In particular, if we recall that the elementary symmetric polynomials $P_{\ell, k}$ of the $k$ variables $y^{1}, \ldots, y^{k}$ uniquely identify $\left\langle y^{1}, \ldots, y^{k}\right\rangle \in(\mathbb{C})_{\text {sym }}^{k}$, just as the power sums

$$
S_{\ell, k}=\sum_{j=0}^{k}\left(y_{j}\right)^{\ell}
$$

do, we have the following Lemma.

Lemma 2.5. A point $\alpha=\left\langle\alpha^{1}, \ldots, \alpha^{k}\right\rangle \in\left(\mathbb{C}^{n}\right)_{\text {sym }}^{k}$ is uniquely determined by each of the following:

i) $\varphi_{\ell, \gamma}\left(\alpha^{1}, \ldots, \alpha^{k}\right)$ for $0 \leq \ell \leq k,|\gamma|=\ell$;

ii) $S_{\beta}\left(\alpha^{1}, \ldots, \alpha^{k}\right)=\sum_{j=1}^{k}\left(\alpha^{j}\right)^{\beta} 0 \leq|\beta| \leq \ell$.

Proof. Let us start with i). If we know the $\varphi_{\ell, \gamma}$ for $|\gamma|=\ell, 0 \leq \ell \leq k$, we know $P_{\ell, k}\left(u \cdot \alpha^{1}, \ldots, u \cdot \alpha^{k}\right)$ for $0 \leq \ell \leq k$ and every $u \in \mathbb{C}^{n}$. Hence, we know $\left\langle u \cdot \alpha^{1}, \ldots, u \cdot \alpha^{k}\right\rangle \in(\mathbb{C})_{\text {sym }}^{k}$ for every $u \in \mathbb{C}^{n}$, and thus, $\left\langle\alpha^{1}, \ldots, \alpha^{k}\right\rangle \in\left(\mathbb{C}^{n}\right)_{\text {sym }}^{k}$. 
For ii), if we know $S_{\beta}\left(\alpha^{1}, \ldots, \alpha^{k}\right)$ for $|\beta| \leq k$, we know $S_{\ell, k}\left(u \cdot \alpha^{1}, \ldots, u \cdot \alpha^{k}\right)$ for $0 \leq \ell \leq k$ and every $u \in \mathbb{C}^{n}$, and as before, this means we know $\left\langle u \cdot \alpha^{1}, \ldots, u \cdot \alpha^{k}\right\rangle \in$ $(\mathbb{C})_{\text {sym }}^{k}$ for every $u \in \mathbb{C}^{n}$, and therefore also $\left\langle\alpha^{1}, \ldots, \alpha^{k}\right\rangle \in\left(\mathbb{C}^{n}\right)_{\text {sym }}^{k}$.

If we look at the polynomial

$$
P_{n, k}(u \cdot z, u)=\sum_{|\gamma|=m} \Phi_{\gamma}(z, \xi) u^{\gamma},
$$

the $\Phi_{\gamma}$ are polynomials in $z$ with coefficients which are holomorphic in $\xi \in \Delta_{\xi}$. The equations

$$
\Phi_{\gamma}(z, \xi)=0,|\gamma|=k
$$

define $\mathcal{X}$ in $\Delta_{z} \times \Delta_{\xi}$ and will be referred to as the standard defining equations of $\mathcal{X}$. Note that

$$
\Phi_{\gamma}(z, \xi)=\frac{k !}{\gamma !} z^{\gamma}+\sum_{\beta<\gamma} a_{\beta}^{\gamma}(\xi) z^{\beta} .
$$

Example 2.6. The Bishop surface $w=\lambda\left(z^{2}+\bar{z}^{2}\right)+|z|^{2}$ is a germ of a Segrenondegenerate hypersurface in $\mathbb{C}^{2}$ of multiplicity 2 if $\lambda \neq 0$. Its standard defining equations are given by

$$
\begin{aligned}
& \Phi_{(2,0)}(z, w, \bar{z}, \bar{w})=\left(-\frac{\bar{w}}{\lambda}+\frac{|z|^{2}}{\lambda}+\bar{z}^{2}+z^{2}\right), \\
& \Phi_{(1,1)}(z, w, \bar{z}, \bar{w})=\frac{(w-\bar{w})(\bar{z}+2 \lambda z)}{\lambda} \\
& \Phi_{(0,2)}(z, w, \bar{z}, \bar{w})=(w-\bar{w})^{2} .
\end{aligned}
$$

We finally note that by Proposition 2.3 we can also consider the "barred" defining equations (in a possibly smaller polydisc), where $z$ and $\xi$ change their roles, that is, the functions

$$
\overline{\Phi_{\gamma}}(\xi, z), \quad|\gamma|=k,
$$

are also (standard) defining equations for $\mathcal{X}$ (considered as the complexification of $(X, 0)$ in a possibly smaller neighbourhood of the origin).

2.2. The Segre varieties as multifunctions. Given $(X, 0) \subset \mathbb{C}^{n}$, the Segre variety $S_{q}$ of a point $\bar{q} \in \tilde{\Delta}_{\xi}$ with respect to the $\pi_{2}$-good polydiscs $\tilde{\Delta}_{z} \times \tilde{\Delta}_{\xi}$ is usually defined by

$$
S_{q}=\left\{z \in \tilde{\Delta}_{z}:(z, \bar{q}) \in \mathcal{X}\right\},
$$

where $\mathcal{X}$ is, as before, the complexification of $(X, 0)$ with respect to $\tilde{\Delta}_{z} \times \tilde{\Delta}_{\xi}$. For generic $q$, we have that $\left|S_{q}\right|=k$. If we would like the map $q \mapsto S_{q}$ to be antiholomorphic, we need to identify it with the holomorphic multifunction $Z: \tilde{\Delta}_{\xi} \rightarrow\left(\tilde{\Delta}_{z}\right)_{\mathrm{sym}}^{k}$ defined by

$$
Z(\xi)=\left\langle z^{1}(\xi), \ldots, z^{k}(\xi)\right\rangle, \quad \xi \in \tilde{\Delta}_{\xi},
$$

where $Z(\xi)=\left(\pi_{2} \mid \mathcal{X}\right)^{-1}(\xi)$ via $S_{q}=Z(\bar{q})$. We can equivalently consider $\Xi: \Delta_{z} \rightarrow$ $\left(\Delta_{\xi}\right)_{\mathrm{sym}}^{k}$,

$$
\Xi(z)=\left\langle\xi^{1}(z), \ldots, \xi^{k}(z)\right\rangle, \quad z \in \Delta_{z}
$$

where $\Xi(z)=\left(\pi_{1} \mid \mathcal{X}\right)^{-1}(z)$ for suitable polydiscs $\Delta_{z} \times \Delta_{\xi}$; by Proposition 2.3, these multifunctions (as germs at 0 ) are related by $Z=\bar{\Xi}$ (or equivalently $\Xi=\bar{Z}$, where we define the barred multifunction by $\overline{Z(\xi)}=\bar{Z}(\bar{\xi})$. 
By Proposition 2.3 these two maps have additional properties which are usual for Segre varieties. We combine this with the basic invariance result for holomorphic maps: note that if $H:\left(\mathbb{C}^{n}, 0\right) \rightarrow\left(\mathbb{C}^{n^{\prime}}, 0\right)$ is a germ of a holomorphic map satisfying $H((X, 0)) \subset(Y, 0)$, then the map $\mathcal{H}(z, \xi)=(H(z), \bar{H}(\xi))$ satisfies $\mathcal{H}((\mathcal{X}, 0) \subset$ $(\mathcal{Y}, 0)$, where $\mathcal{Y}$ denotes the complexification of $(Y, 0)$.

Lemma 2.7. Let $(X, 0) \subset\left(\mathbb{C}^{n}, 0\right),(Y, 0) \subset\left(\mathbb{C}^{n^{\prime}}, 0\right)$ be germs of Segre-nondegenerate real-analytic varieties at the origin, $\tilde{\Delta}_{z} \times \tilde{\Delta}_{\xi}$ be $\pi_{2}$-good for $(X, 0)$, and $\tilde{\Delta}_{z^{\prime}} \times \tilde{\Delta}_{\xi^{\prime}}$ be $\pi_{2}^{\prime}$-good for $(Y, 0)$. Also, let $H:\left(\mathbb{C}^{n}, 0\right) \rightarrow\left(\mathbb{C}^{n^{\prime}}, 0\right)$ be a germ of a holomorphic map satisfying $H((X, 0)) \subset(Y, 0)$. Let $Z$ and $Z^{\prime}$ be defined as above. Then the following hold (provided all of the expressions involved are defined, and the good polydiscs are small enough):

i) $p \in Z(\bar{q})$ if and only if $q \in Z(\bar{p})$.

ii) $z \in Z(\bar{z})$ if and only if $z \in(X, 0)$.

iii) $H(p) \in Z^{\prime}(\overline{H(q)})$ if $p \in Z(\bar{q})$.

Proof. The content of the Lemma are just convenient restatements of the fact that $Z(\xi)=\pi_{z}^{-1}(\xi)$ (and similarly for $Z^{\prime}$ ). So in order to prove i), $p \in Z(\bar{q})$ means that $(p, \bar{q}) \in \mathcal{X}$, which in turn means that $(q, \bar{p}) \in \mathcal{X}$, i.e. $q \in Z(\bar{p})$ (provided the latter is defined). ii) follows similarily.

For iii), we appeal to the fact that $\mathcal{H}((\mathcal{X}, 0)) \subset(\mathcal{Y}, 0)$, which means that $p \in Z(\bar{q})$ implies $(H(p), \overline{H(q)}) \in \mathcal{Y}$, i.e. $H(p) \in Z^{\prime}(\overline{H(q)})$.

2.3. Real-analytic and formal functions on $(X, 0)$. A function $f: X \cap U \rightarrow \mathbb{C}$ is said to be real-analytic on $X \cap U$ if there exists a neighbourhood $\tilde{U}$ of $X$ in $\mathbb{C}^{n}$ and a real-analytic function $\tilde{f}: \tilde{U} \rightarrow \mathbb{C}$ on $\mathbb{C}^{n}$ such that $\left.\tilde{f}\right|_{X \cap U}=f$. The $\operatorname{set} \mathcal{C}^{\omega}(X, 0)$ of germs of real-analytic functions on $X$ at 0 is therefore naturally identified with the local function ring of $X$ at 0 , which we are going to denote by

$$
\mathbb{C}\{X\}=\mathbb{C}\{z, \bar{z}\} / \mathcal{I}_{0}(X)
$$

We also define the ring of formal functions of $X$ by

$$
\mathbb{C} \llbracket X \rrbracket=\mathbb{C} \llbracket z, \bar{z} \rrbracket / \hat{\mathcal{I}}_{0}(X),
$$

where $\hat{\mathcal{I}}_{0}(X)=\mathbb{C} \llbracket z, \bar{z} \rrbracket \mathcal{I}_{0}(X)$ is the ideal generated by $\mathcal{I}_{0}(X) \subset \mathbb{C} \llbracket z, \bar{z} \rrbracket$ in the ring of germs of formal power series. Note that $\hat{\mathcal{I}}_{0}(X)$ is again a real ideal. The corresponding rings in the complexification are

$$
\mathbb{C}\{\mathcal{X}\}=\mathbb{C}\{z, \xi\} / \mathcal{I}_{0}(\mathcal{X}), \quad \mathbb{C} \llbracket \mathcal{X} \rrbracket=\mathbb{C} \llbracket z, \bar{z} \rrbracket / \hat{\mathcal{I}}_{0}(\mathcal{X}) \cdot
$$

Note that the rings $\mathbb{C}\{\mathcal{X}\}$ and $\mathbb{C}\{X\}$ as well as $\mathbb{C} \llbracket \mathcal{X} \rrbracket$ and $\mathbb{C} \llbracket X \rrbracket$ are isomorphic; however, it is convenient to distinguish between $X$ and its complexification $\mathcal{X}$.

An important remark, echoing Proposition 2.4 in the formal setting, is that the natural maps

$$
\mathbb{C} \llbracket z \rrbracket \rightarrow \mathbb{C} \llbracket X \rrbracket, \quad \mathbb{C} \llbracket z \rrbracket \rightarrow \mathbb{C} \llbracket \mathcal{X} \rrbracket, \quad \mathbb{C} \llbracket \bar{z} \rrbracket \rightarrow \mathbb{C} \llbracket X \rrbracket, \quad \mathbb{C} \llbracket \xi \rrbracket \rightarrow \mathbb{C} \llbracket X \rrbracket,
$$

are all injections:

Lemma 2.8. If $(X, 0)$ is a Segre-nondegenerate germ, then there are natural inclusions

$$
\mathbb{C} \llbracket z \rrbracket \subset \mathbb{C} \llbracket X \rrbracket, \quad \mathbb{C} \llbracket z \rrbracket \subset \mathbb{C} \llbracket \mathcal{X} \rrbracket, \quad \mathbb{C} \llbracket \bar{z} \rrbracket \subset \mathbb{C} \llbracket X \rrbracket, \quad \mathbb{C} \llbracket \xi \rrbracket \subset \mathbb{C} \llbracket X \rrbracket .
$$


Proof. We only prove this for $\mathbb{C} \llbracket \bar{z} \rrbracket \subset \mathbb{C} \llbracket X \rrbracket$, the other assertion being either direct consequences or analogous to that. Since $X$ is Segre-nondegenerate, if we choose a set of generators $\varrho^{1}, \ldots, \varrho^{d}$ of $\mathcal{I}_{0}(X)$, the map $\mathbb{C}^{n} \ni z \mapsto H(z)=$ $\left(\varrho^{1}(z, 0), \ldots, \varrho^{d}(z, 0)\right) \in \mathbb{C}^{d}$ is finite. Therefore, the matrix $\frac{\partial H}{\partial z}(z)$ is generically of full rank; hence the matrix $\frac{\partial \varrho}{\partial z}(z, \bar{z})$ is also. If now a function of the form $\varphi(\bar{z})$ is congruent to $0 \bmod \mathcal{I}_{0}(X)$, we can write

$$
\varphi(\bar{z})=\sum_{j=1}^{d} \varphi_{j}(z, \bar{z}) \varrho^{j}(z, \bar{z}) .
$$

Taking a derivative with respect to $z$ yields

$$
0=\sum_{j} \varphi_{j}(z, \bar{z}) \varrho_{z}^{j}(z, \bar{z})+\sum_{j} \varphi_{j, z}(z, \bar{z}) \varrho^{j}(z, \bar{z}),
$$

and so (since the $\varrho_{z}^{j}(z, 0)$ are generically independent) we have that $\varphi_{j}(z, 0)=0$.

Now assume that we know that $\varphi_{j, \bar{z}^{\alpha}}(z, 0)=0$ for $|\alpha|<k$. We take a $\beta \in \mathbb{N}^{n}$ with $|\beta|=k$ and compute

$$
\frac{\partial^{|\beta|} \varphi}{\partial \bar{z}^{\beta}}=\sum_{j} \varphi_{j, \bar{z}^{\beta}} \varrho^{j}+P\left(\varphi_{j, \bar{z}^{\alpha}}:|\alpha|<k\right) .
$$

Taking the derivative with respect to $z$ once again and evaluating at $\bar{z}=0$ yields $\varphi_{j, \bar{z}^{\beta}}(z, 0)=0$. By induction, we therefore have $\varphi_{j}(z, \bar{z})=0, j=1, \ldots, d$, and hence $\varphi=0$.

2.4. Remark: Formal varieties. One can, instead of working with ideals coming from an actual manifold $X$, often also obtain results which are valid for formal Segre-nondegenerate varieties:

Definition 2.9. A radical ideal $\hat{\mathcal{I}} \subset \mathbb{C} \llbracket z, \bar{z} \rrbracket$ is said to define a formal Segre nondegenerate variety $\hat{X}$ (at 0 ) if

(1) $\hat{\mathcal{I}}$ is real, i.e. $\sigma(\hat{\mathcal{I}}) \subset \hat{\mathcal{I}}$, and

(2) $S=\{\varrho(z, 0): \varrho \in \hat{I}\}$ is an ideal of definition, i.e. there exists a $k$ such that the maximal ideal $\hat{\mathfrak{m}} \subset \mathbb{C} \llbracket z, \bar{z} \rrbracket$ satisfies $\hat{\mathfrak{m}}^{k} \subset \hat{\mathcal{I}}$.

The Segre multiplicity of the formal variety $\hat{X}$ is defined to be $\operatorname{dim}_{\mathbb{C}} \mathbb{C} \llbracket z \rrbracket / S$.

We will use the same notation for formal varieties $\hat{X}$ (defined by the ideal $\hat{\mathcal{I}}_{0}(\hat{X})$ ) that we introduced real-analytic varieties above. In particular, we would like to point out that Lemma 2.8 holds (with the same proof) in this setting: i.e. we have that

$$
\mathbb{C} \llbracket z \rrbracket \subset \mathbb{C} \llbracket \hat{X} \rrbracket, \quad \mathbb{C} \llbracket z \rrbracket \subset \mathbb{C} \llbracket \hat{\mathcal{X}} \rrbracket, \quad \mathbb{C} \llbracket z \bar{z} \subset \mathbb{C} \llbracket \hat{X} \rrbracket, \quad \mathbb{C} \llbracket \xi \rrbracket \subset \mathbb{C} \llbracket \hat{X} \rrbracket .
$$

\section{Obstructions to holomorphicity}

It is well known that for a real-analytic maximally real submanifold $E$, every real-analytic function on $E$ is the restriction of a holomorphic function in a neighbourhood of $E$. In the presence of a singularity, this is not necessarily the case any longer, as the following simple example shows. 
Example 3.1. Consider the variety $X$ in $\mathbb{C}_{(z, w)}^{2}$ defined by

$$
z^{3}=w^{2}+\bar{w}^{2} .
$$

This variety is Segre-nondegenerate, of Segre multiplicity 6 , and one checks that the real-analytic function $\left.\bar{w}\right|_{X}$ is not the restriction of any holomorphic function in $\mathbb{C}^{2}$ to $X$. Indeed, if it were, say $\left.\bar{w}\right|_{X}=f(z, w)$, then by Proposition 2.4 we would have $f(z, w)^{2}=z^{3}-w^{2}$ as germs at the origin, which is absurd.

However, the Segre multiplicity of $(X, 0)$ gives a rough bound for how many nonholomorphic real-analytic functions there are.

Proposition 3.2. Let $(\hat{X}, 0) \subset\left(\mathbb{C}^{n}, 0\right)$ be a formal Segre nondegenerate subvariety, of Segre multiplicity $k$. Then there exists an operator

$$
T: \mathbb{C} \llbracket z, \bar{z} \rrbracket \rightarrow \mathbb{C} \llbracket z \rrbracket[\bar{z}]
$$

valued in the space of polynomials of degree at most $k$ in $\bar{z}$ such that for every formal power series $f \in \mathbb{C} \llbracket z, \bar{z} \rrbracket$, we have that $T f(z, \bar{z}) \in \mathbb{C} \llbracket z \rrbracket[\bar{z}]$ is a representative for the class of $f$ in $\mathbb{C} \llbracket \hat{X} \rrbracket$. Furthermore, if $X$ is a real-analytic variety and if $f \in \mathbb{C}\{X\}$, then $T f \in \mathbb{C}\{z, \bar{z}\}$.

Proof. We use the standard defining equations introduced in (11) above in their conjugate versions in an adaptation of standard Weierstrass division. So let

$$
\bar{\Phi}_{\gamma}(\bar{z}, z)=\frac{k !}{\gamma !} \bar{z}^{\gamma}+\sum_{\beta<\gamma} a_{\beta}^{\gamma}(z) \bar{z}^{\beta} \in \hat{\mathcal{I}}_{0}(\hat{X}),
$$

where the $a_{\beta}^{\gamma}(z) \in \mathbb{C} \llbracket z \rrbracket$ vanish at 0 . For any formal power series $\varphi(z, \bar{z})$, we write

$$
\varphi(z, \bar{z})=\varphi_{0}(z, \bar{z})+\sum_{|\gamma|=k} \bar{z}^{\gamma} T_{\gamma} \varphi(z, \bar{z})
$$

with $\varphi_{0}(z, \bar{z})$ a polynomial of degree at most $k$ in $\bar{z}$ and some choice of $T_{\gamma}$. Consider the operator $S: \mathbb{C} \llbracket z, \bar{z} \rrbracket \rightarrow \mathbb{C} \llbracket z, \bar{z} \rrbracket$ defined by

$$
(S \varphi)(z, \bar{z})=\varphi_{0}(z, \bar{z})+\sum_{|\gamma|=k} \frac{\gamma !}{k !} \bar{\Phi}_{\gamma}(\bar{z}, z) T_{\gamma} \varphi(z, \bar{z}) .
$$

Then $(I-S) \varphi$ vanishes to order strictly exceeding the order of vanishing of $\varphi$ at 0 . It follows that $S$ is bijective, its inverse given by $S^{-1} f=\sum_{j}(I-S)^{j} f$. Given a formal power series $f(z, \bar{z})$, denote by $\varphi(z, \bar{z})=\left(S^{-1} f\right)(z, \bar{z})$. We then have

$$
f(z, \bar{z})=(S \varphi)(z, \bar{z})=\varphi_{0}(z, \bar{z})+\sum_{|\gamma|=k} \frac{\gamma !}{k !} \bar{\Phi}_{\gamma}(\bar{z}, z) T_{\gamma} \varphi(z, \bar{z}) .
$$

Therefore, $f(z, \bar{z})$ and $\varphi_{0}$ (which is a polynomial of degree at most $z$ ) agree modulo the standard defining equations of $\hat{X}$.

For the proof of convergence, we need a little preparation, even though it is very similar. In that case, $X$ is given by real-analytic equations, and hence the $a_{\beta}^{\gamma}(z)$ converge in a neighbourhood of the origin. We denote by $\Delta_{r}^{p}$ and $\Delta_{s}^{q}$ two polydiscs (of arbitrary polyradius $r$ and $s$, respectively) in $\mathbb{C}^{p}$ and $\mathbb{C}^{q}$, respectively. We first prove the following 
Claim: For $p, q$, and $k \in \mathbb{N}$, there exist operators $T_{\gamma}: \mathcal{H}^{\infty}\left(\Delta_{r}^{p} \times \Delta_{s}^{q}\right)$ for $\gamma \in \mathbb{N}^{q}$ with $|\gamma|=k$ such that

$$
\varphi(z, w)=\varphi_{0}(z, w)+\sum_{|\gamma|=k} w^{\gamma} T_{\gamma} \varphi(z, w)
$$

where $\varphi_{0}(z, w)$ is a polynomial of degree less than $k$ in $w$, and such that with $\|\cdot\|_{K}$ denoting the supremum norm over a set $K$,

$$
\left\|T_{\gamma} \varphi(z, w)\right\|_{\Delta_{r}^{p} \times \Delta_{s}^{q}} \leq\left(\frac{2}{s}\right)^{\gamma}\|\varphi(z, w)\|_{\Delta_{r}^{p} \times \Delta_{s}^{q}} .
$$

The proof of this claim is by induction on $q$. For $n=1$, we write

$$
\varphi\left(z, w_{1}\right)=\varphi(z, 0)+w_{1}(T \varphi)(z, w),
$$

where $T \varphi\left(z, w_{1}\right)=\frac{\varphi\left(z, w_{1}\right)-\varphi(z, 0)}{w_{1}}$ clearly satisfies

$$
\|T \varphi\|_{\Delta_{r} \times \Delta_{s}} \leq \frac{2}{s_{1}}\|\varphi\|_{\Delta_{r} \times \Delta_{s}} .
$$

We can thus write

$$
\varphi\left(z, w_{1}\right)=\sum_{j=0}^{k-1} \varphi_{j}(z) w_{1}^{j}+w_{1}^{k}\left(T^{k} \varphi\right)\left(z, w_{1}\right),
$$

with

$$
\left\|\varphi_{j}\right\|_{\Delta_{r}^{p} \times \Delta_{s_{1}}} \leq\left(\frac{2}{s_{1}}\right)^{j}\|\varphi\|_{\Delta_{r}^{p} \times \Delta_{s_{1}}}, \quad\left\|\left(T^{k} \varphi\right)\left(z, w_{1}\right)\right\|_{\Delta_{r}^{p}} \leq\left(\frac{2}{s_{1}}\right)^{k}\|\varphi\|_{\Delta_{r}^{p} \times \Delta_{s_{1}}} .
$$

Assuming that we know the corresponding estimates in dimension $q-1$, we write $w=\left(w^{\prime}, w_{n}\right) \in \mathbb{C}^{n-1} \times \mathbb{C}$ and (as before in the one variable case) obtain

$$
\varphi(z, w)=\sum_{j=0}^{k-1} \varphi_{j}\left(z, w^{\prime}\right) w_{n}^{j}+w_{n}^{k}\left(T^{k} \varphi\right)(z, w),
$$

with

$$
\left\|\varphi_{j}\right\|_{\Delta_{r}^{p} \times \Delta_{s^{\prime}}^{q-1}} \leq\left(\frac{2}{s_{n}}\right)^{j}\|\varphi\|_{\Delta_{r}^{p} \times \Delta_{s}^{q}}, \quad\left\|\left(T^{k} \varphi\right)\left(z, w_{1}\right)\right\|_{\Delta_{r}^{p} \times \Delta_{s}^{q}} \leq\left(\frac{2}{s}\right)^{k}\|\varphi\|_{\Delta_{r}^{p} \times \Delta_{s}^{q}} .
$$

We thus define $T_{(0, \ldots, 0, k)}:=T^{k}$ and for $\ell<k$,

$$
T_{\left(\gamma^{\prime}, \ell\right)} \varphi(z, w)=T_{\gamma^{\prime}} \varphi_{\ell}\left(z, w^{\prime}\right),
$$

which satisfies all of our requirements.

With the claim, we can now set up the operator $S$ as in the formal part of the proof, but as an operator between $\mathcal{H}^{\infty}\left(\Delta_{r}^{n} \times \Delta_{s}^{n}\right)$, for small $(r, \ldots, r)$ and $(s, \ldots, s)$. When estimating $I-S$, one now obtains that

$$
\|(I-S) \varphi\|_{\Delta_{r}^{n} \times \Delta_{s}^{n}} \leq C \frac{\max _{\gamma, \beta}\left\|a_{\gamma}^{\beta}\right\|_{\Delta_{r}^{p}}}{s^{k}}
$$

which for small $r$ is small, and thus, for such $r$ this operator is invertible (as an endomorphism of the Banach space $\left.\mathcal{H}^{\infty}\left(\Delta_{r}^{n} \times \Delta_{s}^{n}\right)\right)$. The rest of the proof is then exactly as before. 
Recall from $\S 2.4$ that $\mathbb{C} \llbracket z \rrbracket \subset \mathbb{C} \llbracket \hat{X} \rrbracket$. The preceding Proposition says in particular that

is finite.

$$
\operatorname{dim}_{\mathbb{C}} \mathbb{C} \llbracket \hat{X} \rrbracket / \mathbb{C} \llbracket z \rrbracket \leq\left(\begin{array}{c}
n+k-1 \\
k-1
\end{array}\right)
$$

The next theorem characterizes holomorphy of a germ $f(z, \bar{z})$ in the sense that a real-analytic function on a real-analytic Segre nondegenerate variety comes from the restriction of a holomorphic function if (and only if) its complexification is constant along the fibers of the projection on the first coordinate.

Theorem 3.3. Let $(X, 0) \subset\left(\mathbb{C}^{n}, 0\right)$ be an irreducible germ of a Segre nondegenerate $n$-dimensional real-analytic subvariety. Let $f \in \mathcal{C}^{\omega}(X, 0)$ be represented by $f(z, \bar{z}) \in$ $\mathbb{C}\{z, \bar{z}\}$, so that $f$ is holomorphic on $\Delta_{z} \times \Delta_{\xi}$, which we assume to be $\pi_{1}$-good for $(X, 0)$. Denote by $\mathcal{X} \subset \Delta_{z} \times \Delta_{\xi}$ the corresponding complexification. Then there exists a holomorphic function $F: \Delta_{z} \rightarrow \mathbb{C}$ such that $f(z, \bar{z})=F(z)$ for $z \in X$ close by the origin if and only if there exists an open set $U \subset \Delta_{z}$ such that

$$
f\left(z, \xi_{1}\right)=f\left(z, \xi_{2}\right)
$$

whenever $z \in U$ and $\left(z, \xi_{1}\right) \in \mathcal{X}$ and $\left(z, \xi_{2}\right) \in \mathcal{X}$.

Proof. We only need to prove the "if" part. As $\mathcal{X}$ is irreducible, then the regular part $\mathcal{X}_{\text {reg }}$ is connected. Suppose $\pi_{1}$ is generically $k$ to 1 . Let $V \subset \mathcal{X}_{\text {reg }}$ be the set where $\pi_{1}$ is $k$ to 1 , that is $V=\mathcal{X}_{\text {reg }} \backslash \pi_{1}^{-1}(D)$ where $D$ is the discriminant set of the projection, a complex analytic subvariety. Taking two preimages $\xi_{1}$ and $\xi_{2}$ as functions of $z$, then for any $z_{0} \in \Delta_{z} \backslash D$ we can analytically continue $f\left(z, \xi_{1}(z)\right)-$ $f\left(z, \xi_{2}(z)\right)$ until we get to a $z \in U$. As $U$ is an open set and $f\left(z, \xi_{1}(z)\right)-f\left(z, \xi_{2}(z)\right)$ is identically zero on $U$, we find that $f\left(z_{0}, \xi_{1}\left(z_{0}\right)\right)-f\left(z_{0}, \xi_{2}\left(z_{0}\right)\right)$. Or in other words, $f\left(z, \xi_{1}\right)=f\left(z, \xi_{2}\right)$ whenever $z \in \Delta_{z} \backslash D$ and $\left(z, \xi_{1}\right)$ and $\left(z, \xi_{2}\right)$ are in $\mathcal{X}$.

Thus for all $z \in \Delta_{z} \backslash D$, define $F(z)=f(z, \xi)$ for some $\xi$ such that $(z, \xi) \in \mathcal{X}$. Clearly $F$ is well defined by the above argument. $F$ is locally bounded as $f$ is locally bounded in all of $\Delta_{z} \times \Delta_{\xi}$. Further $D$ is a subvariety of $\Delta_{z}$, and so by the Riemann extension theorem, $F$ is a holomorphic function on $\Delta_{z}$.

In the next section we will introduce the "right" formulation of the preceding Theorem in order to be able to make it into a formal statement as well.

\section{The averaging operator}

Let $(X, 0) \subset\left(\mathbb{C}^{n}, 0\right)$ be an irreducible germ at 0 of Segre-nondegenerate $n$ dimensional subvariety, of Segre multiplicity $k$. Let $\Delta_{z} \times \Delta_{\xi}$ be good for $(X, 0)$, and let $\mathcal{X} \subset \Delta_{z} \times \Delta_{\xi}$ be the corresponding complexification.

In the previous section we proved that a function on $X$ that complexifies to $\Delta_{z} \times \Delta_{\xi}$ is a restriction of a holomorphic function if and only if $\xi \mapsto f(z, \xi)$ is constant on $\left(\pi_{1} \mid \mathcal{X}\right)^{-1}(z)$. We may therefore average out an arbitrary real analytic function to obtain a holomorphic function as follows.

We recall the multifunctions $Z(\xi)$ and $\Xi(z)$, which are defined near the origin with values in $\left(\Delta_{z}\right)_{\mathrm{sym}}^{k}$ and $\tilde{\Delta}_{\xi}$, respectively, by (4) and (5). Given a germ of a realanalytic function $f \in \mathbb{C}\{z, \bar{z}\}$, assume that $f$ extends to be a holomorphic function on $\Delta \times \bar{\Delta}$, so that

$$
g\left(z,\left\langle\omega^{1}, \ldots, \omega^{k}\right\rangle\right)=\frac{1}{k} \sum_{j=1}^{k} f\left(z, \omega^{j}\right),
$$


is a holomorphic function on $\Delta \times(\bar{\Delta})_{\text {sym }}^{k}$. For a suitable neighbourhood polydisc $\tilde{\Delta} \subset \Delta$, we can assume that $\Xi(\tilde{\Delta}) \subset(\bar{\Delta})_{\text {sym }}^{k}$.

Hence we can define a holomorphic function $\mathcal{A} f: \tilde{\Delta} \rightarrow \mathbb{C}$ by

$$
(\mathcal{A} f)(z)=g(z, \Xi(z))=\frac{1}{k} \sum_{j=1}^{k} f\left(z, \xi^{j}(z)\right)
$$

This definition gives a (linear) map $\mathcal{A}: \mathbb{C}\{z, \bar{z}\} \rightarrow \mathbb{C}\{z\}$, and the reader can check that $\mathcal{A}: \mathbb{C} \llbracket z, \bar{z} \rrbracket \rightarrow \mathbb{C} \llbracket z \rrbracket$ can be defined for formal power series, even if $\hat{X}$ is merely assumed to be formal.

The next result summarizes the properties which show that the operator $\mathcal{A}$ encodes the obstruction to holomorphic extension of a real-analytic function on $(X, 0)$.

Lemma 4.1. Let $(X, 0)$ be a germ of a real-analytic Segre nondegenerate variety.

The mapping $\mathcal{A}$ has $\mathcal{I}_{0}(X) \subset \operatorname{ker} \mathcal{A}$. In particular, it descends to a map (again denoted by the same letter $\mathcal{A}: \mathcal{C}^{\omega}(X, 0) \rightarrow \mathbb{C}\{z\}$.

A function $f \in \mathcal{C}^{\omega}(X, 0)$ is the restriction of a germ of a holomorphic function on $\mathbb{C}^{n}$ if and only if $\left.\mathcal{A} f\right|_{X}=\left.f\right|_{X}$.

Proof. We have already discussed linearity. If $\varrho \in \mathbb{C}\{z, \bar{z}\}$ vanishes on $(X, 0)$, its complexification vanishes on $\mathcal{X}$, and hence $\mathcal{A} \varrho=0$. For the last statement, we only need to prove the necessity of the given characterization. By Theorem 3.3. $f\left(z, \xi_{1}\right)=f\left(z, \xi_{2}\right)$ when $\left(z, \xi_{1}\right),\left(z, \xi_{2}\right) \in \mathcal{X}$. It follows that $\left.\mathcal{A} f\right|_{X}=\left.f\right|_{X}$.

We note that together with Proposition 3.2, this gives a rather complete picture of the obstructions to holomorphicity: they are encoded in the behaviour of $\mathcal{A}$ on functions of the form $\bar{z}^{\alpha}$ for $|\alpha|<k$. Before we discuss this fact further, we give some examples.

Example 4.2. We again consider the Bishop surface $w=\lambda\left(z^{2}+\bar{z}^{2}\right)+z \bar{z}$. The function $\Xi(z)$ is computed to be

$\Xi(z)=\left\langle\left(\frac{-z+\sqrt{-4 \lambda^{2} z^{2}+z^{2}+4 w \lambda}}{2 \lambda}, w\right),\left(\frac{-z-\sqrt{-4 \lambda^{2} z^{2}+z^{2}+4 w \lambda}}{2 \lambda}, w\right)\right\rangle$.

One therefore computes that

$$
\mathcal{A} \bar{z}=\frac{-z}{2 \lambda}, \quad \mathcal{A} \bar{w}=w
$$

Note that the computation of

$$
\mathcal{A} \bar{z}^{2}=\frac{2 \lambda w+\left(1-2 \lambda^{2}\right) z^{2}}{\lambda^{2}} .
$$

can be done using the defining relation of the surface as well as direct application of the definition of the averaging operator. We will return to this observation in more generality below.

First, the averaging operator depends only on values on $X$, which is clear since $\mathcal{A} f$ is holomorphic, but we have more. The following proposition replaces equality of two real-analytic functions on $(X, 0)$ by equality of two germs of holomorphic functions $\left(\mathbb{C}^{n}, 0\right)$. In particular, since $\mathcal{A}$ is defined canonically, we no longer need to consider the defining functions of $X$. Furthermore this equality can now be done formally. 
Proposition 4.3. Suppose $(X, 0) \subset \mathbb{C}^{n}$ is an irreducible germ of a Segre nondegenerate $n$-dimensional real-analytic subvariety of multiplicity $k$. Let $f$ and $g$ be germs of real-analytic functions. Then $\left.f\right|_{X}=\left.g\right|_{X}$ if and only if $\mathcal{A}\left((f-g)^{\ell}\right)=0$ for all $\ell=1, \ldots, k$.

Proof. If $\left.f\right|_{X}=\left.g\right|_{X}$, then also their complexifications are equal on the complexification of $X$. For a point on $X, \mathcal{A} f$ only depends on the values of $f$ on the complexification of $X$, and so $\mathcal{A} f=\mathcal{A} g$; the same argument holds for all powers of $f$ and $g$.

On the other hand suppose $\mathcal{A}\left((f-g)^{\ell}\right)=0$ for all $\ell=1, \ldots, k$. Consider a good neighbourhood $\Delta_{z} \times \Delta_{\xi}$ for $X$. We can assume that $X$ is a closed subset of $\Delta_{z}$. Fix a point $z \in X$. By the definition of the averaging operator and the hypothesis of the proposition,

$$
\sum_{j=1}^{k}\left(f\left(z, \xi^{j}(z)\right)-g\left(z, \xi^{j}(z)\right)\right)^{\ell}=0
$$

The power sums for powers 1 through $k$ uniquely determine an unordered set of $k$ complex numbers. Hence it must be that $f\left(z, \xi^{j}(z)\right)=g\left(z, \xi^{j}(z)\right)$ for all $j$. Since $z \in X$, then for at least one $j$, we have $\xi_{j}(z)=\bar{z}$ and therefore, $f(z, \bar{z})=g(z, \bar{z})$.

Similarly, we can check if a function is a restriction of a holomorphic function.

Theorem 4.4. Suppose $(X, 0) \subset \mathbb{C}^{n}$ is an irreducible germ of a Segre nondegenerate $n$-dimensional real-analytic subvariety of multiplicity $k$. Suppose that $f(z, \bar{z})$ is a real-analytic function. Then $\left.f\right|_{X} \in \mathbb{C}\{z\}$ if and only if $\mathcal{A}\left(f^{\ell}\right)=(\mathcal{A} f)^{\ell}$ for all $\ell=1, \ldots, k$.

Proof. If $\left.f\right|_{X}=\left.h\right|_{X}$ for a holomorphic $h$, then $\left.f^{\ell}\right|_{X}=\left.h^{\ell}\right|_{X}$ for all $\ell$ and therefore $\mathcal{A}\left(f^{\ell}\right)=h^{\ell}=\mathcal{A}(f)^{\ell}$ for all $\ell$. Then

On the other hand, suppose $\mathcal{A}\left(f^{\ell}\right)=(\mathcal{A} f)^{\ell}$ for all $\ell=1, \ldots, k$ and let $h=\mathcal{A} f$.

$$
\mathcal{A}(f-h)^{\ell}=\sum_{j=0}^{\ell}(-1)^{j}\left(\begin{array}{l}
\ell \\
j
\end{array}\right) h^{\ell-j} \mathcal{A}\left(f^{j}\right)=0,
$$

and so by Proposition 4.3, $f=\mathcal{A} f$, i.e. $f$ is holomorphic.

\section{Flattening AND Other APPlichtions of AVERAGing}

Even though we introduced the averaging operator for arbitrary real-analytic function germs on $(X, 0)$, it is completely determined by its action on the subspace $\mathbb{C}\{\bar{z}\} \subset \mathcal{C}^{\omega}(X, 0)$. We therefore define the restricted averaging operator

$$
\mathcal{R}=\left.\mathcal{A}\right|_{\mathbb{C}\{\bar{z}\}}: \mathbb{C}\{\bar{z}\} \rightarrow \mathbb{C}\{z\} .
$$

The operator $\mathcal{A}$ can be recovered from $\mathcal{R}$ since

$$
\mathcal{A}\left(z^{\alpha} \bar{z}^{\beta}\right)=z^{\alpha} \mathcal{A}\left(\bar{z}^{\beta}\right)=z^{\alpha} \mathcal{R}\left(\bar{z}^{\beta}\right) .
$$

We can use the restricted averaging operator to characterize another important property of a real-analytic variety $(X, 0)$, namely, whether it is flattenable. We will say that $(X, 0)$ can be flattened if there exists a germ of a holomorphic function $f(z) \in \mathbb{C}\{z\}$ such that $\left.f\right|_{X}$ has real values. We shall write $\bar{f} \in \mathbb{C}\{\bar{z}\}$ for the antiholomorphic function defined by $\bar{f}(\bar{z})=\overline{f(z)}$. The terminology is explained by the fact that $(X, 0)$ can be flattened if and only if there exists a (possibly 
singular) Levi flat hypersurface, defined by $\operatorname{Im} f=0$, containing $(X, 0)$. Also being flattenable is encoded in the averaging operator.

Theorem 5.1. Suppose $(X, 0) \subset \mathbb{C}^{n}$ is an irreducible germ of a Segre nondegenerate $n$-dimensional real-analytic subvariety of multiplicity $k$. Suppose that $f(z, \bar{z})$ is a real-analytic function. Then a holomorphic function $f$ is real-valued on $X$ (that is, $X$ is flattenable) if and only if $\mathcal{R}\left(\bar{f}^{\ell}\right)=f^{\ell}$ for all $\ell=1, \ldots, k$.

Proof. If $f$ is a holomorphic function that is real-valued on $X$, then $\left.\bar{f}^{\ell}\right|_{X}=\left.f^{\ell}\right|_{X}$ for all $\ell$. Hence $\mathcal{R}\left(\bar{f}^{\ell}\right)=\mathcal{R}\left(f^{\ell}\right)=f^{\ell}$ for all $\ell$.

On the other hand, suppose that $\mathcal{R}\left(\bar{f}^{\ell}\right)=f^{\ell}$ for all $\ell=1, \ldots, k$. We can then compute that

$$
\mathcal{A}(f-\bar{f})^{\ell}=\sum_{j=0}^{\ell}(-1)^{j}\left(\begin{array}{l}
\ell \\
j
\end{array}\right) f^{j} \mathcal{R}\left(\bar{f}^{\ell-j}\right)=0 .
$$

As a final application of the restricted averaging operator $\mathcal{R}$, we note that it contains all the information necessary to define $X$.

Theorem 5.2. Let $M_{1}, M_{2}$ be germs of Segre-nondegenerate real-analytic subvarieties of multiplicity $k$, and let $\mathcal{R}_{1}, \mathcal{R}_{2}$ be their restricted averaging operators. If $\mathcal{R}_{1} \bar{z}^{\beta}=\mathcal{R}_{2} \bar{z}^{\beta}$ for every $\beta$ with $|\beta| \leq k$, then $M_{1}=M_{2}$.

Proof. We claim that given the restricted averaging operator of any such variety $M$ allows us to reconstruct the defining equation. The restricted averaging operator gives us the power sums of all monomials $\bar{z}^{\beta}$ for $|\beta| \leq k$, from which we can explicitly compute the elementary symmetric functions (see e.g. [16]) needed to construct the standard defining equations (11). Thus, $M$ is uniquely determined by its restricted averaging operator.

\section{EXAMPLES OF FLATTENING}

We are now going to consider in some detail models of the form

$$
M_{p}: w=p(z, \bar{z})=\sum_{j} \alpha_{j} z^{j} \bar{z}^{k-j},
$$

in particular, for the cases $k=2,3$. These were considered by Moser-Webster [18] for $k=2$ and Harris [7 for $k \geq 2$ before. Harris showed that one can consider them as the lowest order invariant (in a suitable sense) at a CR singular point of a codimension 2 submanifold of $\mathbb{C}^{2}$.

We shall, for our purposes, introduce weights $k$ for $w$ and 1 for $z$ and use a bit of a different normalization than Harris did, adapted in particular to the case that we are interested in, namely, that $M_{p}$ is Segre nondegenerate, which means that $p(z, 0) \neq \equiv$ in our setting.

This allows us to choose coordinates in such a way that $\alpha_{k}=0$ and $\alpha_{0}=1$. This fixes coordinates $(z, w)$ for $M_{p}$ up to a finite group of rotations in $z$, unless $p(z, \bar{z})=\bar{z}^{2 k}+\alpha_{k}|z|^{2 k}$; in that case, we can normalize further so that $\alpha_{k} \geq 0$.

The averaging operator for $M_{p}$ will be denoted by $\mathcal{A}_{p}$, and the restricted averaging operator for $M_{p}$ will be denoted by $\mathcal{R}_{p}$. We also denote the space of (weighted) bihomogeneous polynomials of degree $a$ in $(z, w)$ and $b$ in $(\bar{z}, \bar{w})$ by

$$
\mathcal{P}_{a, b}:=\left\{p \in \mathbb{C}[z, w, \bar{z}, \bar{w}]: p\left(t z, t^{k} w, s \bar{z}, s^{k} \bar{w}\right)=t^{a} s^{b} p(z, w, \bar{z}, \bar{w})\right\} .
$$


We then have

Lemma 6.1. The averaging operator $\mathcal{A}_{p}$ maps weighted homogeneous polynomials to weighted homogeneous polyonomials:

$$
\mathcal{A}_{p}: \mathcal{P}_{a, b} \rightarrow \mathcal{P}_{a+b, 0}, \quad \mathcal{R}_{p}: \mathcal{P}_{0, b} \rightarrow \mathcal{P}_{b, 0} .
$$

Proof. It is enough to show that the restricted averaging operator satisfies the claim. Denoting as usual by $\xi^{1}(z, w), \ldots, \xi^{k}(z, w)$ the points $\left(z, w, \xi^{j}(z, w)\right) \in \mathcal{X}$, where the complexification is now defined on all of $\mathbb{C}^{4}$, we write $\xi^{j}=\left(\zeta^{j}, \eta^{j}\right)$ and first find that the elementary symmetric functions $s^{1}, \ldots, s^{k}$ of the $\zeta^{j}$ satisfy

$$
s^{k}\left(\zeta^{1}(z, w), \ldots, \zeta^{k}(z, w)\right)=-w, \quad s^{j}\left(\zeta^{1}(z, w), \ldots, \zeta^{k}(z, w)\right)=\alpha_{j} z^{j} .
$$

If we now compute the restricted averaging operator, we have

$$
\begin{aligned}
\mathcal{R}\left(\bar{z}^{r} \bar{w}^{s}\right) & =\mathcal{R}\left(\bar{z}^{r} \bar{p}(\bar{z}, z)^{s}\right) \\
& =\mathcal{R}\left(\sum_{\ell=s}^{k s} A_{\ell} \bar{z}^{r+k s-\ell} z^{\ell}\right) \\
& =\sum_{\ell=s}^{k s} A_{\ell}\left(\frac{1}{k} \sum_{j=1}^{k}\left(\zeta^{j}(z, w)\right)^{r+k s-\ell} z^{\ell}\right) .
\end{aligned}
$$

Now each of the power sums $\frac{1}{k} \sum_{j=1}^{k}\left(\zeta^{j}(z, w)\right)^{r+k s-\ell}$ is homogeneous (in $z$ and $w$ ) of degree $r+k s-\ell$, because by the basic theorem on symmetric polynomials, we can rewrite the power sum as a polynomial of the form

$$
\begin{aligned}
\frac{1}{k} \sum_{j=1}^{k}\left(\zeta^{j}(z, w)\right)^{r+k s-\ell} & =S\left(s^{1}(\zeta(z, w)), \ldots, s^{k}(\zeta(z, w))\right) \\
& =S\left(\alpha_{1} z, \alpha_{2} z^{2}, \ldots, \alpha_{k-1} z^{k-1},-w\right)
\end{aligned}
$$

where $S\left(x_{1}, \ldots, x_{k}\right)$ is weighted homogeneous of degree $r+k s-\ell$, when $x_{j}$ has weight $j$. The claim follows.

The representation of a real-analytic function as a polynomial in $(\bar{z}, \bar{w})$ with holomorphic coefficients is also very simple on our models.

Lemma 6.2. Every real-analytic germ $f \in \mathcal{C}^{\omega}\left(M_{p}, 0\right)$ can be written uniquely in the form

$$
f(z, w, \bar{z}, \bar{w})=\sum_{j=0}^{k-1} f_{j}(z, w) \bar{z}^{j} .
$$

Proof. We use Weierstrass division: First, we divide $f(z, w, \bar{z}, \bar{w})$ by $\bar{w}-\bar{p}(\bar{z}, z)$, which yields

$$
f(z, w, \bar{z}, \bar{w})=(\bar{w}-\bar{p}(\bar{z}, z)) q(z, w, \bar{z}, \bar{w})+r(z, w, \bar{z}),
$$

and then the remainder $r$ by $w-p(z, \bar{z})$, regarded as a $k$-regular function in $\bar{z}$ :

$$
r(z, w, \bar{z})=(w-p(z, \bar{z})) \tilde{q}(z, w, \bar{z})+\sum_{j=0}^{k-1} f_{j}(z, w) \bar{z}^{j} .
$$

For the last part of the statement, apply Theorem 4.1 
Let us remark that the product of two such representations can be computed quite efficiently, since one can use the defining equation

$$
\bar{z}^{k}+\alpha_{1} \bar{z}^{k-1} z+\cdots+\alpha_{k-1} \bar{z} z^{k-1}-w=0
$$

to express $\bar{z}^{j}$ for $j \geq k$ recursively.

An adaptation of that idea is the basis for the proof of the following:

Lemma 6.3. The restricted averaging operator satisfies

$$
R\left(\bar{z}^{a} \bar{w}^{b}\right)= \begin{cases}O(z) & b \geq 1 \text { or } a \neq 0 \quad \bmod k, \\ w^{\frac{a}{k}}+O(z) & b=0 \text { and } a \cong 0 \quad \bmod k .\end{cases}
$$

Proof. Recalling that $M_{p}$ is given by $w=p(z, \bar{z})$ or equivalently by $\bar{w}=\bar{p}(\bar{z}, z)$, where

$$
p(z, \bar{z})=\bar{z}^{k}+\sum_{j=1}^{k-1} \alpha_{j} z^{j} \bar{z}^{k-j},
$$

we have that

$$
\bar{z}^{a} s^{a} w=\sum_{j} \alpha_{j} z^{j} \bar{z}^{k-j+a} s^{a} .
$$

Applying $\mathcal{R}$ and summing over $a \in \mathbb{N}$, we write

$$
R(s)=\sum_{a=0}^{\infty} \mathcal{R}\left(\bar{z}^{a}\right) s^{a}, \quad R_{c}(s)=\sum_{\substack{a \\ a<c}} \mathcal{R}\left(\bar{z}^{a}\right) s^{a},
$$

and obtain

$$
R(s) w=\sum_{j=0}^{k-1} \alpha_{j} z^{j} \frac{R(s)-R_{k-j}(s)}{s^{k-j}}
$$

so that

$$
R(s)=\frac{\sum_{j} \alpha_{j} z^{j} s^{j-k} R_{k-j}(s)}{p\left(z, s^{-1}\right)-w}=\frac{1+O(z)}{1+O(z)-s^{k} w} .
$$

This proves the claim for $b=0$. For $b \geq 1$, we see from the defining equation of $M_{p}$ that $\bar{w}=O(z)$, so that $R\left(\bar{z}^{a} \bar{w}^{b}\right)=O(z)$ also.

Example 6.4. Let us discuss the standard quadric in our context. Its defining equation will be written as

$$
w=\bar{z}^{2}+2 \mu z \bar{z}, \quad 0 \leq \mu<\infty
$$

(we use $\alpha_{1}=2 \mu$ because it simplifies some of the formulas which follow). The associated $\zeta^{1}, \zeta^{2}$ are given by

$$
\zeta^{1}(z, w)=-\mu z+\sqrt{w+\mu^{2} z^{2}}, \quad \zeta^{2}(z, w)=-\mu z-\sqrt{w+\mu^{2} z^{2}} .
$$

The restricted averaging operator is given by

$$
\mathcal{R} \bar{z}=-\mu z, \quad \mathcal{R} \bar{w}=\left(1-2 \mu^{2}\right) z^{2},
$$


In the case of the quadric, we can actually also give formulas for $\mathcal{R}$ based on

$$
\begin{aligned}
\mathcal{R} \bar{z}^{j} & =\frac{1}{2}\left(\left(-\mu z+\sqrt{w+\mu^{2} z^{2}}\right)^{j}+\left(-\mu z-\sqrt{w+\mu^{2} z^{2}}\right)^{j}\right) \\
& =(-1)^{j} \sum_{p=0}^{\lfloor j / 2\rfloor}\left(\begin{array}{c}
j \\
2 p
\end{array}\right)(\mu z)^{j-2 p}\left(w+\mu^{2} z^{2}\right)^{p} \\
& =(-1)^{j} \sum_{p=0}^{\lfloor j / 2\rfloor} \sum_{q=0}^{p}\left(\begin{array}{c}
j \\
2 p
\end{array}\right)\left(\begin{array}{l}
p \\
q
\end{array}\right)(\mu z)^{j-2 p+2 q} w^{p-q} ;
\end{aligned}
$$

the formulas for $\mathcal{R} \bar{z}^{j} \bar{w}^{k}$ can be worked out similarly. We also note that the generating function $R(s)$ from above is given explicitly by

$$
R(s)=\frac{1-\mu z+2 \mu z s}{1+2 \mu z s-w} .
$$

The real-valued formal holomorphic maps on a model manifold can be explicitly described as follows.

Theorem 6.5. Assume that $f \in \mathbb{C} \llbracket z, w \rrbracket$ is real-valued (but not constant) on $M_{p}$, and that $p(z, \bar{z}) \neq \bar{z}^{k}$. Then there exists a unique $\vartheta \in \mathbb{R}$ such that $e^{i \vartheta} p(z, \bar{z})+$ $e^{-i \vartheta} z^{k}=e^{-i \vartheta} \bar{p}(\bar{z}, z)+e^{i \vartheta} \bar{z}^{k}$ and

$$
f(z, w)=\sum_{j=0}^{\infty} f_{j}\left(e^{i \vartheta} w+e^{-i \vartheta} z^{k}\right)^{j}, \quad f_{j} \in \mathbb{R} .
$$

Proof. We first check that it is enough to determine the weighted homogeneous polynomials $P(z, w)$ with the property that $\bar{P}(\bar{z}, \bar{w})=P(z, w)$ on $M$, and to show that these need to be of the form $P(z, w)=f_{j}\left(w+z^{k}\right)^{j}$. Indeed, if we know the statement for the polynomials, then given any $f \in \mathbb{C} \llbracket z, w \rrbracket$, we decompose $f=\sum_{j=j_{0}}^{\infty} P_{j}$ with $P_{j_{0}} \neq 0 . \quad f$ being real-valued implies that $\mathcal{R}\left(\bar{f}^{a}\right)=f^{a}$ for $a=1, \ldots, k$, so that $\mathcal{R}\left(\bar{P}_{j_{0}}^{a}\right)=P_{j_{0}}^{a}$ for $a=1, \ldots, k$ and thus $P_{j_{0}}$ is real valued on $M$. The series $\tilde{f}=f-P_{j_{0}}$ is therefore of higher vanishing order than $f$ and still real valued. Inductively, we can rewrite $f$ as a sum of real-valued homogeneous polynomials.

By homogeneity reasons, for $1 \leq j<k$, every homogeneous polynomial of degree $j$ is necessarily a polynomial of $z$ and therefore never real-valued.

For a homogeneous polynomial of degree $k$, we have $P_{k}(z, w)=a z^{k}+b w$, which restricted to $M_{p}$ becomes

$$
P_{k}(z, p(z))=a z^{k}+b \bar{z}^{k}+\sum_{j=1} b \alpha_{j} z^{j} \bar{z}^{k-j} .
$$

Hence $P_{k}$ is real-valued on $M_{p}$ if and only if $a=\bar{b}$ and $\bar{b} \bar{\alpha}_{j}=b \alpha_{k-j}$ for $j=$ $1, \ldots, k-1$. By assumption, at least one of the $\alpha_{j}$ is not zero, so that we can find the $\vartheta$ in the polar decomposition $b=f_{k} e^{-i \vartheta}$ from $p$ alone. Hence, we have $P_{k}=f_{k}\left(e^{i \vartheta} w+e^{-i \vartheta} z^{k}\right)$ as claimed.

We now proceed by induction on the degree $j$ of $P$, and assume that we have proved our claim for all weighted homogeneous polynomials of lesser degree. After dividing by $\left(e^{i \vartheta} w+e^{-i \vartheta} z^{k}\right)$, we have that

$$
P(z, w)=\left(e^{i \vartheta} w+e^{-i \vartheta} z^{k}\right) q(z, w)+r(z),
$$


with $q$ weighted homogeneous of degree $j-k$. Since $P=\bar{P}$ on $M$, we have that

$$
\left(e^{i \vartheta} w+e^{-i \vartheta} z^{k}\right)(q(z, w)-\overline{q(z, w)})=\overline{r(z)}-r(z)
$$

on $M$. Now both the left and the right hand side of this equation are imaginaryvalued on $M$. One checks as before that this means that $r=0$. It follows that $q(z, w)=\overline{q(z, w)}$, and so the induction hypothesis applies to $q$; if $q$ is of weighted degree not divisible by $k$, this means that $q=0$, while if $q$ is of weighted degree divisible by $k, q$ is a real multiple of $\left(e^{i \vartheta} w+e^{-i \vartheta} z^{k}\right)^{j}$.

\section{REFERENCES}

[1] Patrick Ahern and Xianghong Gong, Real analytic manifolds in $\mathbb{C}^{n}$ with parabolic complex tangents along a submanifold of codimension one, Ann. Fac. Sci. Toulouse Math. (6) 18 (2009), no. 1, 1-64. MR2518102

[2] Errett Bishop, Differentiable manifolds in complex Euclidean space, Duke Math. J. 32 (1965), 1-21. MR200476

[3] Adam Coffman, Unfolding CR singularities, Mem. Amer. Math. Soc. 205 (2010), no. 962, viii+90. MR2650710

[4] Xiang Hong Gong, On the convergence of normalizations of real analytic surfaces near hyperbolic complex tangents, Comment. Math. Helv. 69 (1994), no. 4, 549-574. MR1303227

[5] Xianghong Gong and Laurent Stolovitch, Real submanifolds of maximum complex tangent space at a CR singular point, I, Invent. Math. 206 (2016), no. 2, 293-377. MR3570294

[6] _ Real submanifolds of maximum complex tangent space at a CR singular point, II, J. Differential Geom. 112 (2019), no. 1, 121-198. MR3948229

[7] Gary A. Harris, Lowest order invariants for real-analytic surfaces in $\mathbf{C}^{2}$, Trans. Amer. Math. Soc. 288 (1985), no. 1, 413-422. MR773068

[8] Gary Alvin Harris, The traces of holomorphic functions on real submanifolds, Trans. Amer. Math. Soc. 242 (1978), 205-223. MR477120

[9] Xiao Jun Huang and Steven G. Krantz, On a problem of Moser, Duke Math. J. 78 (1995), no. $1,213-228$. MR1328757

[10] Xiaojun Huang, On an n-manifold in $\mathbf{C}^{n}$ near an elliptic complex tangent, J. Amer. Math. Soc. 11 (1998), no. 3, 669-692. MR1603854

[11] Geometric and analytic problems for a real submanifold in $\mathbb{C}^{n}$ with $C R$ singularities, Sci. China Math. 60 (2017), no. 6, 995-1004. MR3647129

[12] Xiaojun Huang and Wanke Yin, A Bishop surface with a vanishing Bishop invariant, Invent. Math. 176 (2009), no. 3, 461-520. MR2501295

[13] Carlos E. Kenig and Sidney M. Webster, The local hull of holomorphy of a surface in the space of two complex variables, Invent. Math. 67 (1982), no. 1, 1-21. MR664323

[14] - On the hull of holomorphy of an n-manifold in $\mathbf{C}^{n}$, Ann. Scuola Norm. Sup. Pisa Cl. Sci. (4) 11 (1984), no. 2, 261-280. MR764946

[15] Jiří Lebl, Alan Noell, and Sivaguru Ravisankar, Extension of CR functions from boundaries in $\mathbb{C}^{n} \times \mathbb{R}$, Indiana Univ. Math. J. 66 (2017), no. 3, 901-925. MR3663330

[16] I. G. Macdonald, Symmetric functions and Hall polynomials, Second, Oxford Classic Texts in the Physical Sciences, The Clarendon Press, Oxford University Press, New York, 2015. With contribution by A. V. Zelevinsky and a foreword by Richard Stanley, Reprint of the 2008 paperback edition [ MR1354144]. MR3443860

[17] Jürgen Moser, Analytic surfaces in $\mathbf{C}^{2}$ and their local hull of holomorphy, Ann. Acad. Sci. Fenn. Ser. A I Math. 10 (1985), 397-410. MR802502

[18] Jürgen K. Moser and Sidney M. Webster, Normal forms for real surfaces in $\mathbf{C}^{2}$ near complex tangents and hyperbolic surface transformations, Acta Math. 150 (1983), no. 3-4, 255-296. MR709143

[19] S. M. Webster, The Euler and Pontrjagin numbers of an $n$-manifold in $\mathbf{C}^{n}$, Comment. Math. Helv. 60 (1985), no. 2, 193-216. MR800003

[20] Hassler Whitney, Complex analytic varieties, Addison-Wesley Publishing Co., Reading, Mass.-London-Don Mills, Ont., 1972. MR0387634 